\section{Sequential Chemilumi- nescent Detection of Target DNAs without Stripping and Reprobing}

BioTechniques 26:710-714 (April 1999)

\begin{abstract}
We present a simple method for sequential chemiluminescent detections of two different DNA loci on a single Southern blot. First, an enzyme-linked DNA probe for a unique sequence is detected with a horseradish peroxidase (HRP) substrate followed by the detection of another enzyme-linked DNA probe for a different unique sequence with an alkaline phosphatase (AP) substrate that simultaneously inhibits the chemiluminescence generated by HRP. Such sequential detection steps eliminate the need to strip and reprobe blots and can be performed with no intervening steps.
\end{abstract}

\section{INTRODUCTION}

The chemiluminescent detection of target DNA, besides being safer and as sensitive as radioisotopic detection, offers versatility in the labeling of probes and choice of detection formats. In a commonly used format, probes are labeled with haptens such as biotin, fluorescein or digoxigenin (Dig). The hapten is then bound with its corresponding ligand or antibody conjugated with a reporter enzyme such as horseradish peroxidase (HRP) or alkaline phosphatase (AP). The reporter enzyme is detected with a chemiluminescent substrate.

Recognizing that blotting analyses are labor-intensive and time-consuming, techniques have been developed to increase the amount of information that can efficiently be obtained from a blot, e.g., the stripping of probes and rehybridization of the blot with additional probes $(4,9)$. For example, genetic fingerprinting of forensic DNA samples by restriction fragment-length polymorphism (RFLP) analysis requires analyzing a Southern blot with several probes. Often the limited availability of
DNA necessitates the stripping and reprobing of the same blot multiple times $(1,7)$. In northern blot analysis where the expression of a specific gene is measured at the messenger RNA (mRNA) level, the signal is normalized by reprobing the blot for $\alpha$-tubulin or $\beta$-actin mRNAs. Detection steps in western blotting can also involve stripping off antibodies from the initial round of hybridization and hybridizing the blot with another set of antibodies for additional data (5).

Potential savings in time, effort and sample amount afforded by stripping and reprobing is gained at the expense of membrane-bound target nucleic acids (7) or proteins (5) that are lost causing reduced detection sensitivities in the second and subsequent detection steps. In this report, we present an alternate strategy for sequentially detecting two membrane-bound targets that avoids the disadvantages of stripping and reprobing. This method is demonstrated in an assay of two different DNA analytes. The assay format consists of simultaneously hybridizing two differently labeled probes that bind to corresponding ligands or antibodies (one conjugated with HRP and the other with AP), thus enabling the detection of the two reporter enzymes by sequential application of two chemiluminescent reagents. The first reagent contains a peroxidase substrate that emits chemiluminescence from the HRP-labeled bands. The second reagent contains both a phosphatase substrate for detecting the AP-labeled bands and components that stop light emission from the HRP-substrate reaction.

\section{MATERIALS AND METHODS}

\section{Chemiluminescent Substrates}

The chemiluminescent HRP detection reagent Lumigen ${ }^{\mathrm{TM}}$ PS-3 was obtained from Lumigen (Southfield, MI, USA). Chemiluminescent AP detection reagents were prepared by adding various quantities of $30 \% \mathrm{H}_{2} \mathrm{O}_{2}$ (Sigma, St. Louis, MO, USA) to Lumi-Phos ${ }^{\circledR}$ Plus (Lumigen). A concentration of $0.15 \%$ $\mathrm{H}_{2} \mathrm{O}_{2}$ was selected for the method development work. $\mathrm{H}_{2} \mathrm{O}_{2}$-modified Lumi-Phos Plus was prepared by adding $0.5 \mathrm{~mL}$ of $30 \% \mathrm{H}_{2} \mathrm{O}_{2}$ to $100 \mathrm{~mL}$ of Lumi-Phos Plus.

\section{Detection of DNA Markers}

The sequential detection of DNA was first demonstrated using two differently labeled DNA size markers, a biotinylated HindIII enzyme-digested $\lambda$ phage DNA (Life Technologies, Gaithersburg, MD, USA) and a Dig-labeled EcoRI enzyme-digested SPPI marker DNA (DNA Molecular Weight Marker VII ${ }^{2}$; Boehringer Mannheim, Indianapolis, IN, USA). These size markers were fractionated individually in separate lanes and as a mixture in a third lane in a $1 \%$-agarose gel containing ethidium bromide (EtdBr) (Figure 1). The gel was prepared for blotting using the following solutions: (i) depurination $(0.25 \mathrm{M} \mathrm{HCl})$, (ii) denaturing $(0.5 \mathrm{M} \mathrm{NaOH}, 1.5 \mathrm{M} \mathrm{NaCl})$ and (iii) neutralization (0.5 M Tris- $\mathrm{HCl}, \mathrm{pH}$ 7.5, 1.5 M NaCl) and then Southern blotted using a VacuGene ${ }^{\circledR}$ Blotting System (Amersham Pharmacia Bio-

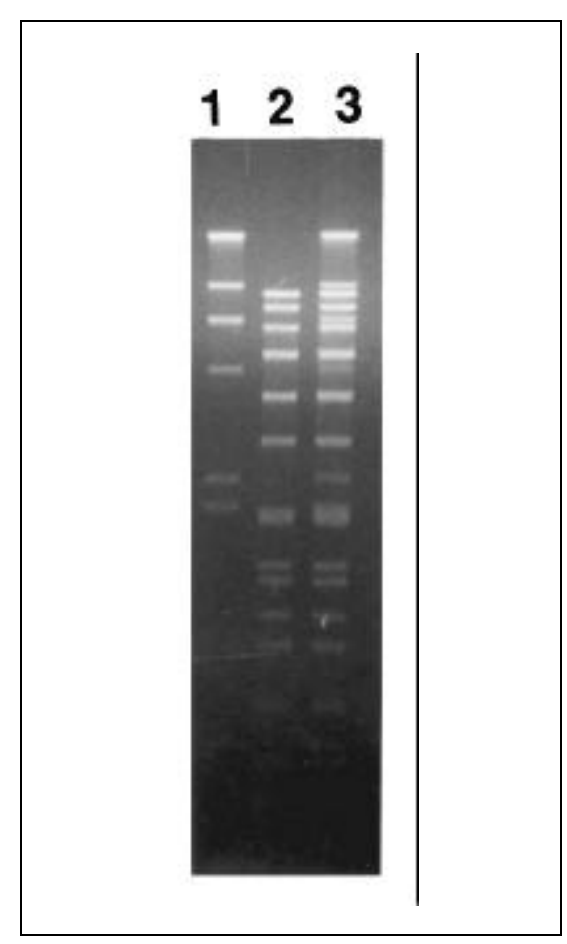

Figure 1. EtdBr-stained 1\% agarose gel containing marker DNA. Lane 1, $200 \mathrm{ng}$ of biotinylated HindIII-digested $\lambda$ DNA; lane 2, $200 \mathrm{ng}$ of Dig-labeled, EcoRI-digested SPP1 size marker DNA; lane 3, $200 \mathrm{ng}$ each of biotinylated HindIII-digested $\lambda \mathrm{DNA}$ and Dig-labeled EcoRI-digested SPP1 size marker DNA. 
tech, Uppsala, Sweden) onto a neutral Hybond ${ }^{\circledR}$-N Nylon Membrane (Amersham Pharmacia Biotech). The blotted membrane was baked at $80^{\circ} \mathrm{C}$ for $2 \mathrm{~h}$.

The blots were first washed for 15 min in $1 \times$ Wash Buffer $(0.1 \mathrm{M}$ maleic acid, $0.15 \mathrm{M} \mathrm{NaCl}, \mathrm{pH} 7.5,0.3 \%$ Tween $^{\circledR} 20$ ) and blocked for $1 \mathrm{~h}$ in $2 \%$ Blocking Buffer (Blocking Reagent [Boehringer Mannheim] dissolved in $0.1 \mathrm{M}$ maleic acid, $0.15 \mathrm{M} \mathrm{NaCl}, \mathrm{pH}$ 7.5). The enzyme conjugates were diluted at 1:5000 in 2\% Blocking Buffer before use. The blots containing the DNA size markers were treated with ImmunoPure ${ }^{\circledR}$ Avidin (Horseradish Peroxidase Conjugated; Pierce Chemical, Rockford, IL, USA) and AntiDigoxigenin-AP (Boehringer Mannheim) enzyme conjugates followed by sequential treatments with the chemiluminescent substrates Lumigen PS-3 for $\mathrm{HRP}$ and $\mathrm{H}_{2} \mathrm{O}_{2}$-modified Lumi-Phos Plus for AP. Both the enzyme and substrate treatments were performed at room temperature; the substrate incu- bations were done in subdued light to minimize exposure of the substrate to bright light. Following the enzymeconjugate treatment, the blots were washed $2 \times$ for 20 min each in $1 \times$ Wash Buffer and then reacted with the HRP substrate for $5 \mathrm{~min}$. Excess substrate was removed by gently pressing the blots in between a pair of transparent plastic sheets, which were then exposed to X-OMAT ${ }^{\circledR}$ AR (XAR) film (Scientific Imaging Systems [Eastman Kodak], New Haven, CT, USA). Exposure times generally ranged in length from a few seconds to minutes for obtaining optimal signal and background. To prepare for the second detection, blots were rinsed for $5 \mathrm{~min}$ each in $1 \times$ Wash Buffer and in AP Detection Buffer (100 $\mathrm{mM}$ Tris- $\mathrm{HCl}, \mathrm{pH} 9.5,100 \mathrm{mM} \mathrm{NaCl}$, $50 \mathrm{mM} \mathrm{MgCl}_{2}$ ) followed by another 5 min treatment with the AP substrate. The blots were then placed between transparent plastic sheets, excess substrate was removed and the signal was captured on XAR film.

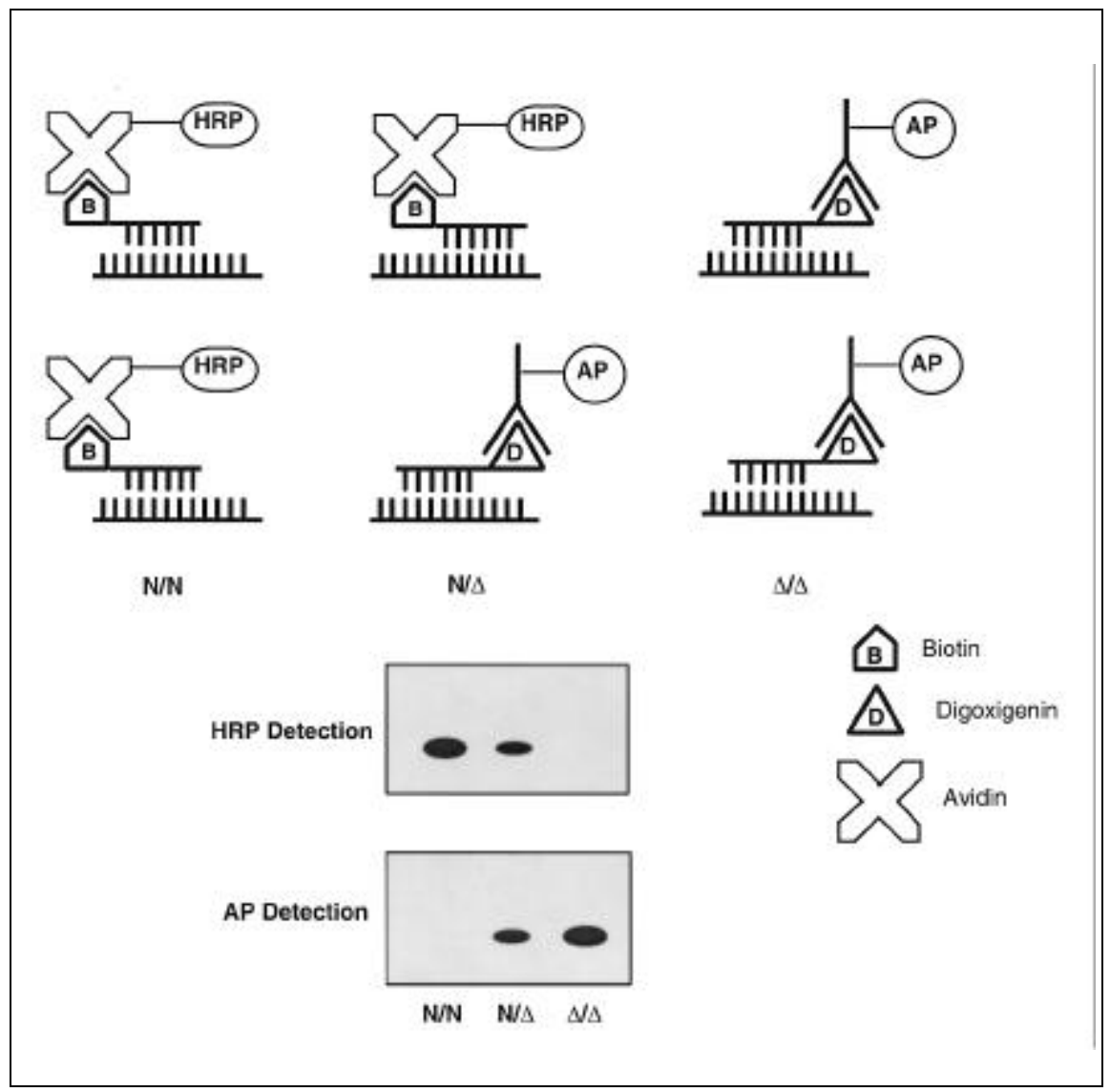

Figure 2. Schematic diagram of the sequential detection of CFTR genotypes with HRP and AP substrates. 


\section{Short Technical Reports}

\section{Southern Blot Analysis of CFTR Genotypes}

Figure 2 shows the sequential detection strategy, as described above, that was applied to detect and differentiate between the genotypes of the cystic fibrosis transmembrane conductance regulator (CFTR) gene with and without the $\Delta \mathrm{F}_{508}$ mutation. The DNA ( 0.5 $\mu \mathrm{g})$ of CFTR genotypes for the $\Delta \mathrm{F}_{508}$ mutation (obtained from Coriell Cell Repositories, Camden, NJ, USA), wildtype $(\mathrm{N} / \mathrm{N})$, heterozygous $\left(\mathrm{N} / \Delta \mathrm{F}_{508}\right)$ and homozygous $\left(\Delta \mathrm{F}_{508} / \Delta \mathrm{F}_{508}\right)$ were amplified by polymerase chain reaction (PCR) using Taq DNA polymerase (8). A pair of PCR primers was designed to amplify the exon 10 region of the CFTR gene containing the mutation (primers were synthesized by Oligos Etc. \& Oligo Therapeutics, Wilsonville, OR, USA). The primers had the sequences: 5'-ACTTCACTTCTAATGATGATTATG-3' and 5'-CTCTTCTAG-
TTGGCATGCTTTGAT-3'. The PCR products were electrophoresed on a $1 \%$ agarose gel and Southern blotted as described above for the DNA size markers. The blot was hybridized simultaneously with a pair of differently labeled (biotin and Dig) oligonucleotide probes in which one was complementary to the normal and the other to the mutant allele. The labeled oligonucleotides were $5^{\prime}$ biotin-ATATCATCTTTGGTGTTTCCT-3' (normal) and 5' Dig-GAAAATATCATTGGTGTTTCC-3' (mutant).

The conditions for prehybridizing and hybridizing the blot were $52^{\circ} \mathrm{C}$ for $1 \mathrm{~h}$ and overnight, respectively, using a $6 \times$ standard saline citrate (SSC); $0.9 \mathrm{M}$ $\mathrm{NaCl}, 0.09 \mathrm{M}$ sodium citrate, $\mathrm{pH}$ 7.0, 0.01 M EDTA, pH 8.0, 5× Denhardt's solution $\left(0.1 \%\right.$ Ficoll $^{\circledR}$ Type 400, 0.1\% polyvinylpyrrolidone, $0.1 \%$ bovine serum albumin), $0.5 \%$ sodium dodecyl sulfate (SDS) and $100 \mu \mathrm{g} / \mathrm{mL}$ denatured salmon sperm DNA (Life Technologies). The post-hybridization

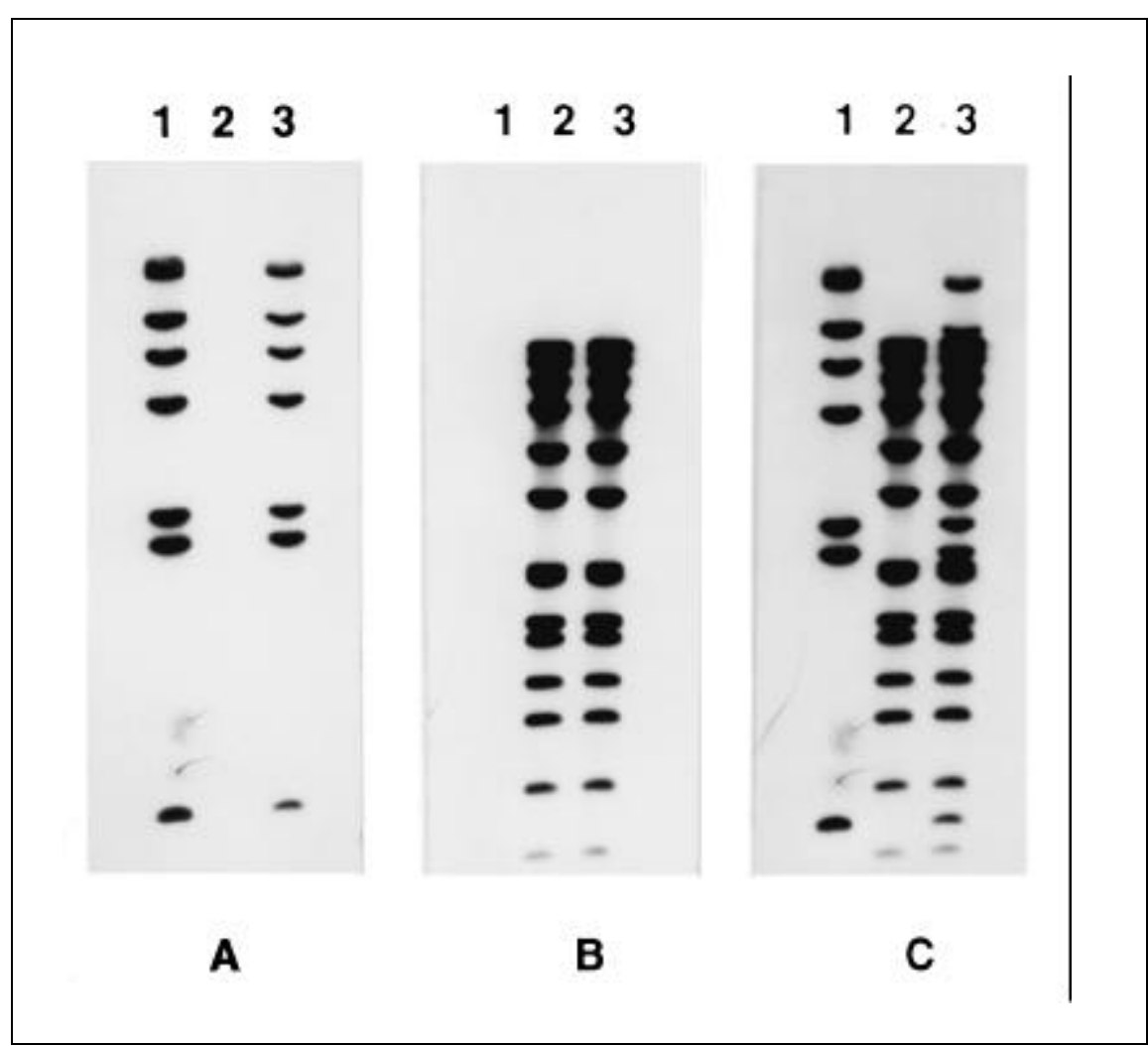

Figure 3. Sequential detection of the blotted DNA size markers shown in Figure 1. (A) HRP substrate (Lumigen PS-3) detection of biotinylated HindIII-digested $\lambda$ DNA. (B) Further treatment of blot with AP substrate $\left(\mathrm{H}_{2} \mathrm{O}_{2}\right.$-modified Lumi-Phos Plus) for the detection of Dig-labeled SPP1 DNA marker. (C) Superimposed picture of the bands in Panels A and B to show that the bands' patterns correspond to those of the EtdBr-stained gel in Figure 1. washes were done at $52^{\circ} \mathrm{C}$ for $20 \mathrm{~min}$ each first in $2 \times \mathrm{SSC}, 0.1 \%$ SDS and then in $0.5 \times$ SSC, $0.1 \%$ SDS. The blots that contained DNA hybridized with the CFTR allele-specific oligonucleotides were treated as described above for washing, blocking and binding of antibodies and detection steps.

\section{RESULTS}

To demonstrate the feasibility of detecting two different DNA sequences by sequential chemiluminescent detection steps, two sets of labeled DNA markers were electrophoresed, blotted onto membranes and bound with enzyme conjugates. One lane contained biotinylated HindIII-digested $\lambda$ DNA, another contained Dig-labeled SPPI-EcoRI size marker DNA and the third lane contained a mixture of both marker DNAs. Incubating the blotted DNA in a solution containing avidin-HRP and antiDig-AP allowed for binding of each enzyme conjugate to its respective ligand. Figure $3 \mathrm{~A}$ shows the resulting image obtained after applying the peroxidase substrate. Figure 3B shows the image

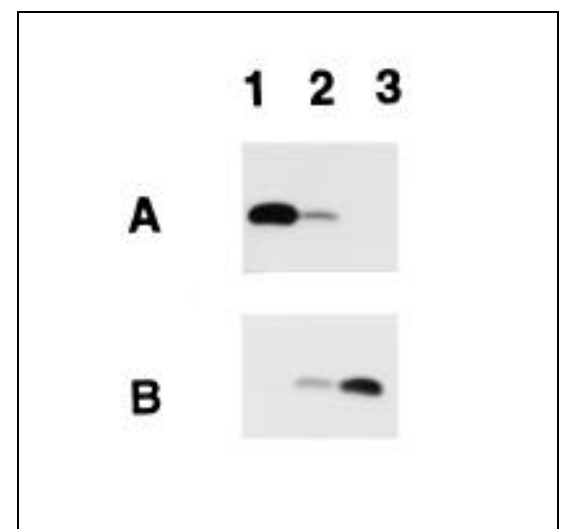

Figure 4. Sequential detection of CFTR genotypes. Southern blot of PCR-amplified sequences from a region of exon 10 of the CFTR gene containing each of the three genotypes. N/N, $\mathrm{N} / \Delta \mathrm{F}_{508}$ and $\Delta \mathrm{F}_{508} / \Delta \mathrm{F}_{508}$ were simultaneously hybridized with pairs of labeled oligonucleotides specific for the normal (biotin labeled) and/or mutant (Dig labeled) alleles and then incubated with avidin-HRP and anti-Dig-AP conjugates. (A) Detection by HRP-generated chemiluminescence of the genotypes containing the normal allele. Lane 1 shows $\mathrm{N} / \mathrm{N}$, whereas lane 2 shows N/ $\Delta$. (B) Detection of the same blot by AP-generated chemiluminescence of the genotypes with the mutant $\Delta \mathrm{F}_{508}$ allele. Lane 2 shows $\mathrm{N} / \Delta$, whereas lane 3 shows $\Delta / \Delta$. 
obtained after subsequent treatment of the same blot with the phosphatase substrate. Upon treatment with Lumigen PS-3, chemiluminescence was generated only from the avidin-HRP bound biotinylated HindIII digested $\lambda$ DNA bands (Figure 3A, lanes 1 and 3 ). The membrane was washed and treated with $\mathrm{H}_{2} \mathrm{O}_{2}$-modified Lumi-Phos Plus, causing the HRP-generated signal to cease and AP-catalyzed chemiluminescence to be produced from the Dig-labeled SPPI-EcoRI size marker in Figure 3B, lanes 2 and 3. Superposition of the images from Figure 3, A and B, produced Figure $3 \mathrm{C}$, which reveals all of the band sizes in lane 3 . These results are consistent with the pattern seen in each of the lanes of the EtdBr-stained agarose gel as shown in Figure 1.

The sequential detection strategy was used for the detection of all three genotypes of the $\mathrm{CF} \Delta \mathrm{F}_{508}$ mutation. PCR-amplified exon 10 regions of the
CFTR gene from each of the three genotypes, N/N, N/ $\Delta \mathrm{F}_{508}$ and $\Delta \mathrm{F}_{508} /$ $\Delta \mathrm{F}_{508}$, were Southern-blotted and simultaneously hybridized with a pair of differently labeled oligonucleotides specific for the normal (biotin labeled) and mutant (Dig labeled) alleles. One incubation step with a mixture of avidin-HRP and anti-Dig-AP conjugates, followed by sequential detections as described above enabled selective detection of the genotypes containing the normal allele, $\mathrm{N} / \mathrm{N}$ and $\mathrm{N} / \Delta$ in the first step (Figure 4A) vs. the genotypes with the mutant $\Delta \mathrm{F}_{508}$ allele, $\mathrm{N} / \Delta$ and $\Delta / \Delta$ (Figure 4B) in the second step.

\section{DISCUSSION}

In most types of applications based on a blotting technique, there is a need to obtain the maximum information with the minimum amount of sample, time and effort. Although stripping and reprobing activities achieve that end to some extent, they are tedious and counterproductive because of the reduction in signal due to membrane-bound template DNA loss during stripping. It is estimated that up to $20 \mathrm{ng}$ of membrane-bound target DNA is lost during each stripping step, depending on the method of probe removal (7). The loss of analyte and the added time and expense of stripping and reprobing can be reduced by a strategy in which the blots are simultaneously hybridized with two differently labeled probes and detected in sequential steps with the two different enzyme substrates of the probes.

The success of the technique described depends on using a pair of enzyme detection reagents in which each can signal the presence of its corresponding enzyme rapidly with no signal arising from the other enzyme. HRP and Lumigen PS-3 were chosen as the 
first enzyme substrate pair because they satisfy several criteria. Rapid and highly sensitive chemiluminescent detection of HRP conjugates in blotting applications has been previously demonstrated using Lumigen PS-3 (2). Moreover the reaction can be rapidly quenched through a fourfold effect when the glowing blot contacts a high $\mathrm{pH}$ buffer that contains a high concentration of peroxide. The alkaline buffer $(\mathrm{pH} \geq 9.5$ ) of Lumi-Phos Plus diminishes peroxidase activity, and the high concentrations of peroxide convert HRP into a catalytically inactive form called HRP compound III (6). In addition, the substrate, 2,3,6-trifluorophenyl 10methylacridine-9-carboxylate is ren- dered nonluminescent by reaction with either hydroxide or hydroperoxide ion.

$\mathrm{AP}$ is one of the most useful enzyme labels because of its high catalytic activity under a variety of conditions including relatively high $\mathrm{pH}$. Inclusion of up to about $0.15 \%(\mathrm{vol} / \mathrm{vol})$ of peroxide in Lumi-Phos Plus had no adverse effect on either the substrate or the enzyme as shown by the generation of chemiluminescence. Since Lumi-Phos Plus has proven to be a robust reagent for use in ultrasensitive blotting applications (3), we chose AP as the second enzyme in the sequential detection scheme and modified Lumi-Phos Plus by addition of $\mathrm{H}_{2} \mathrm{O}_{2}$ for use as the $\mathrm{AP}$ substrate.

Note that after detection of the bound peroxidase label, a brief intermediate wash step with a pH 9.5 buffer was used before wetting the membrane with $\mathrm{H}_{2} \mathrm{O}_{2}$-modified Lumi-Phos Plus. However, the protocol can be simplified and the extra wash step avoided simply by extending the incubation time of the membrane in the second detection reagent to $\geq 15 \mathrm{~min}$ since it has a $\mathrm{pH}$ of 9.6.

We have shown two applications for a sequential chemiluminescent detection method of visualizing two different DNA analytes on a single blot. We have also shown that this strategy can be used to sequentially detect the normal CFTR genotype vs. those harboring the $\Delta \mathrm{F}_{508}$ mutation on a single Southern blot. In other experiments (data not shown), we have used the sequential detection strategy in a genomic Southern blot to detect single copy gene sequences. Even in this assay, which demands greater detection sensitivity, spillover of the first luminescent reaction does not appear to interfere with detection of the second luminescent reaction. While we have illustrated the use of the sequential detection for genotype discrimination, we anticipate that this detection technique can be used in other applications as well.

\section{REFERENCES}

1.Adams, D.E. 1988. Validation of the F.B.I. procedure for DNA analysis: a summary. Crime Lab Digest 15:106-108.

2.Akhavan-Tafti, H., R. DeSilva, Z. Arghavani, K. Sugioka, Y. Sugioka and A.P.
Schaap. 1994. Blotting application of lumigen PS-3 chemiluminescent substrate for horseradish peroxidase, p. 313-316. In A. Campbell, L. Kricka and P. Stanley (Eds.), Bioluminescence and Chemiluminescence Fundamentals and Applied Aspects. John Wiley \& Sons, Chichester.

3.Budowle, B., F.S. Baechtel, C.T. Comey, A.M. Giusti and L. Klevan. 1995. Simple protocols for typing forensic biological evidence: chemiluminescent detection for human DNA quantitation and restriction fragment length polymorphism (RFLP) analyses and manual typing of polymerase chain reaction (PCR) amplified polymorphisms. Electrophoresis 16:1559-1567.

4.Gatti, R.A., P. Concannon and W. Salser. 1984. Multiple use of Southern blots. BioTechniques 2:148-155.

5.Krajewski, S., J.M. Zapata and J.C. Reed. 1996. Detection of multiple antigens on western blots. Anal. Biochem. 236:221-228.

6.Lundin, A. and L. Hallander. 1987. Mechanisms of horseradish peroxidase catalysed luminol reaction in presence and absence of various enhancers, p. 555-558. In J. Schölmerich, R. Andreesen, A. Kapp, M. Ernst and W.G. Woods (Eds.), Bioluminescence and Chemiluminescence New Perspectives. John Wiley \& Sons, Chichester.

7.Noppinger, K., G. Duncan, D. Ferraro, S. Watson and J. Ban. 1992. Evaluation of DNA probe removal from nylon membrane. BioTechniques 13:572-575.

8.Saiki, R.K., D.H. Gelfand, S. Stoffel, S.J. Scharf, R. Higuchi, G.T. Horn, K.B. Mullis and H.A. Erlich. 1988. Primer-directed enzymatic amplification of DNA with a thermostable DNA polymerase. Science 239:487491.

9.Sambrook, J., E.F. Fritsch and T. Maniatis. 1989. Molecular Cloning: A Laboratory Manual, 2nd ed. CSH Laboratory Press, Cold Spring Harbor, NY.

Partial support of this work by SBIR Grant Nos. 2 R44 DK47727-02 and 1 R43 CA75830-01 from the National Institutes of Health is gratefully acknowledged. Address correspondence to Dr. Hashem AkhavanTafti, Lumigen, Inc., 24485 W. Ten Mile Rd., Southfield, MI 48034, USA. Internet: hat@lumigen.com

Received 29 July 1998; accepted 11 January 1999.

L.V. Reddy, R. DeSilva, R.S. Handley, A.P. Schaap and H. Akhavan-Tafti

Lumigen

Southfield, MI, USA 